\title{
WHICH QUATERNION ALGEBRAS ACT ON A MODULAR ABELIAN VARIETY?
}

\author{
VICTOR RotGer
}

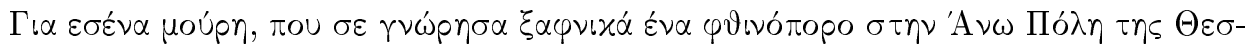
$\sigma \alpha \lambda o v i x \eta s$.

\begin{abstract}
Let $A / \mathbb{Q}$ be a modular abelian variety. We establish criteria to prevent a given quaternion algebra $B$ over a totally real number field to be the endomorphism algebra of $A$ over $\overline{\mathbb{Q}}$. We accomplish this by analyzing the representation of $\operatorname{Gal}(\overline{\mathbb{Q}} / \mathbb{Q})$ on the points of $N$-torsion of $A$ at primes $N$ which ramify in $B$.
\end{abstract}

\section{Introduction}

Let $A / \mathbb{Q}$ be an abelian variety of $\mathrm{GL}_{2}$-type over $\mathbb{Q}$ of dimension $g \geq 1$, by which we mean that the ring of endomorphisms $R=\operatorname{End}_{\mathbb{Q}}(A)$ is an order in a number field $E$ of degree $[E: \mathbb{Q}]=g$.

Let $K / \mathbb{Q}$ be the minimal number field over which all endomorphisms of $A \times \overline{\mathbb{Q}}$ are defined and let $\mathcal{O}=\operatorname{End}_{K}(A)$. Let $B=\operatorname{End}_{K}^{0}(A):=\operatorname{End}_{K}(A) \otimes_{\mathbb{Z}} \mathbb{Q}$.

As a consequence of the recent work on Serre's modularity conjecture by KhareWintenberger [KaWi], Dieulefait [Di] and Kisin [Ki], the generalized Shimura-

Taniyama Conjecture holds true. This amounts to say that $A$ is isogenous over $\mathbb{Q}$ to a factor of the Jacobian variety $J_{1}(L)$ of the modular curve $X_{1}(L)$ for some positive integer $L \geq 1$.

As in $[\mathrm{BFGR}, \S 1]$, christen $(\mathcal{O}, R)$ a modular pair. The minimal level of $(\mathcal{O}, R)$ is the minimal value of $L \geq 1$ as above. Similarly, we shall also say that a triplet $(\mathcal{O}, R, K)$ is modular if $\operatorname{End}_{\mathbb{Q}}(A) \simeq R$ and $\operatorname{End}_{K}(A)=\mathcal{O}$ for some modular abelian variety $A$.

A conjecture attributed to Coleman (cf. [BFGR, §1]) predicts that, for any fixed $g \geq 1$, there exist only finitely many isomorphism classes of modular pairs $(\mathcal{O}, R)$. Also, the possibilities for modular triplets $(\mathcal{O}, R, K)$ should also be very limited. The reader may consult [BFGR, $\S 1]$ for further motivation of this conjecture.

It is the purpose of these notes to address this question for absolutely simple modular abelian varieties $A / \mathbb{Q}$ such that $B \supsetneq E$.

Under this assumption, it follows from Albert's classification of involuting simple algebras and the work of Shimura that $g$ is even and $B$ is a totally indefinite division quaternion algebra over a totally real field $F$ of degree $[F: \mathbb{Q}]=g / 2$. In particular, $E$ is a quadratic extension over $F$ and a maximal subfield of $B$.

Received by the editors February 21, 2007.

2000 Mathematics Subject Classification. 11G18, 14G35.

Key words and phrases. Abelian variety, modular, endomorphism, Galois representation.

Partially supported by Ministerio de Ciencia y Tecnología BFM2003-06768-C02-02. 
It is also known that $E$ is totally real and $K$ is imaginary quadratic (cf. Lemma 2.3 (i), (ii)). Write $E=F(\sqrt{m})$ for some totally positive square-free integral element $m \in R_{F}$. Although we choose $m$ square-free, note that there may exist a non principal ideal $\mathfrak{m}_{0}$ of $F$ such that $\mathfrak{m}_{0}^{2} \mid m R_{F}$.

Write also $K=\mathbb{Q}(\sqrt{-d})$ for some square-free integer $d \geq 1$. By a theorem of Ribet (cf. [Ri2, Theorem 5.6]), the values of $m$ and $d$ are related by the isomorphism

$$
B \simeq\left(\frac{-d, m}{F}\right)
$$

Write $\mathfrak{D}=\operatorname{disc}(B)$ for the reduced discriminant of $B$, that is, the square-free product of the finite ideals of $F$ which ramify in $B$. Since $\left(\frac{-d, m}{F}\right)$ is split at all prime ideals $\mathfrak{N} \nmid 2 d m$, it follows from (1) that

$$
\mathfrak{N} \mid d \text { for all } \mathfrak{N} \mid \mathfrak{D}, \mathfrak{N} \nmid 2 m \text {. }
$$

In order to have a numerical flavour of the issue, let us report on some explicit computations. The table below lists relevant numerical data for all modular pairs $(\mathcal{O}, R)$ of minimal level $L \leq 5400$ such that $\mathfrak{D} \neq(1)$ and $[F: \mathbb{Q}] \leq 4$.

We refer the reader to [BFGR, Prop. 1.3] for $L \leq 7000$ when $F=\mathbb{Q}$; see also [Ha] for explicit details when $L \leq 3000$ and $F=\mathbb{Q}$. When $[F: \mathbb{Q}]=2,3$ or 4 , J. Quer performed the necessary computations.

Column $L$ lists the minimal level of the modular pair. In column $\mathfrak{D}$ we provide the norms $\mathrm{N}_{F / \mathbb{Q}}(\mathfrak{N})$ of the prime ideals $\mathfrak{N} \mid \mathfrak{D}$.

\begin{tabular}{|c|c|c|c|c|c|}
\hline$L$ & {$[F: \mathbb{Q}]$} & $\operatorname{disc}(F)$ & $\mathfrak{D}$ & $\mathrm{N}_{F / \mathbb{Q}}(m)$ & $\operatorname{disc}(K)$ \\
\hline 675 & 1 & 1 & {$[2,3]$} & 2 & -3 \\
\hline 1568 & 1 & 1 & {$[2,3]$} & 3 & -4 \\
\hline 243 & 1 & 1 & {$[2,3]$} & 6 & -3 \\
\hline 2700 & 1 & 1 & {$[2,5]$} & 10 & -3 \\
\hline 1568 & 1 & 1 & {$[2,7]$} & 7 & -4 \\
\hline 3969 & 1 & 1 & {$[3,5]$} & 15 & -7 \\
\hline \hline 1089 & 2 & 5 & {$[9,11]$} & 11 & -3 \\
\hline 2592 & 2 & 33 & {$[2,3]$} & 27 & -4 \\
\hline 3872 & 2 & 5 & {$[4,11]$} & 11 & -4 \\
\hline 3872 & 2 & 5 & {$[4,11]$} & 55 & -4 \\
\hline 4356 & 2 & 5 & {$[5,11]$} & 55 & -3 \\
\hline 4761 & 2 & 41 & {$[2,5]$} & 10 & -3 \\
\hline \hline 2187 & 3 & 81 & {$[3,17]$} & 51 & -3 \\
\hline 2187 & 3 & 81 & {$[3,8]$} & 24 & -3 \\
\hline 3969 & 3 & 321 & {$[3,3]$} & 81 & -7 \\
\hline 4563 & 3 & 1436 & {$[2,3]$} & 6 & -3 \\
\hline \hline 3267 & 4 & 5725 & {$[9,11]$} & 11 & -3 \\
\hline 3267 & 4 & 13525 & {$[5,9]$} & 5 & -3 \\
\hline
\end{tabular}

Table 1. Modular pairs of minimal level $L \leq 5400$ and $[F: \mathbb{Q}] \leq 4$. 
Definition 1.1. The set $\mathcal{N}_{\ell}$ of exceptional prime ideals of $F$ for a given prime $\ell$ is

$$
\mathcal{N}_{\ell}=\left\{\mathfrak{N}: \mathfrak{N} \mid \ell, a^{2}-s \ell \text { or } a^{4}-4 a^{2} \ell+\ell^{2}\right\},
$$

for some $s=0,1,2,3,4$ and some $a \in R_{F}, a \neq \sqrt{s \ell}, \sqrt{2 \ell \pm \sqrt{3} \ell}$, such that $|\tau(a)| \leq$ $2 \sqrt{\ell}$ for all $\tau: F \hookrightarrow \mathbb{R}$.

This is obviously a finite set of prime ideals, rather small for small values of $[F: \mathbb{Q}]$ and $\ell$. When $F=\mathbb{Q}$, we have for instance $\mathcal{N}_{2}=\{2,3,5,7\}$ and $\mathcal{N}_{3}=\{2,3,5,11,23\}$.

Given $\mathcal{O}, R, K$, the results of this note provide necessary conditions for the existence of a modular abelian variety $A / \mathbb{Q}$ such that $\operatorname{End}_{\mathbb{Q}}(A) \simeq R$ and $\operatorname{End}_{K}(A) \simeq \mathcal{O}$. For the sake of clarity and applicability, we gather in the theorem below a simplified version of the results which are obtained throughout the article. See the remaining sections for slightly more general statements.

Below, let $\zeta_{n}$ denote a $n$-th primitive root of 1 . Also, let $\mathcal{O}_{\mathfrak{D}}$ denote a maximal order in the division quaternion algebra $B$ of reduced discriminant $\mathfrak{D} \neq(1)$. Recall that an order $\mathcal{O}$ in $B$ is maximal if and only if $\operatorname{disc}(\mathcal{O})=\mathfrak{D}(\mathrm{cf}$. [Vi, p. 84]).

Theorem 1.2. Let $K$ be an imaginary quadratic field, let $F$ be a totally real number field and let $m \in R_{F}$ be a square-free totally positive element. If $\left(\mathcal{O}_{\mathfrak{D}}, R_{F(\sqrt{m})}, K\right)$ is modular, then

(i) $m R_{F}=\mathfrak{m}_{0}^{2} \cdot \mathfrak{m}$, with $\mathfrak{m}_{0}$ and $\mathfrak{m}$ ideals of $R_{F}, \mathfrak{m} \mid \mathfrak{D}$. If $h(F)=1, \mathfrak{m}_{0}=1$.

(ii) Any $\mathfrak{N} \mid \mathfrak{D}, \mathfrak{N} \nmid 2 m$, lies above a prime $N \equiv 3 \bmod 4$.

(iii) Assume $\mathfrak{D} \nmid 2 m$ and $\mathbb{Q}\left(\zeta_{n}+\zeta_{n}^{-1}\right) \not \subset F$ for $n \neq 1,2,3,4,6$. For any prime $\ell$ such that $\left(\frac{K}{\ell}\right) \neq-1$ and $\sqrt{\ell}, \sqrt{2 \ell}, \sqrt{3 \ell}, \sqrt{2 \ell \pm \sqrt{3} \ell} \notin F$, either

- $\mathfrak{N} \in \mathcal{N}_{\ell}$ for all $\mathfrak{N} \mid \frac{\mathfrak{D}}{(\mathfrak{D}, 2 m)}$, or

- $\left(\frac{-\ell}{\mathfrak{N}}\right) \neq 1$ for all $\mathfrak{N} \mid \mathfrak{D}$.

In the remaining sections of this note we shall develop the necessary machinery to prove this result. Let us now illustrate and describe it in more detail.

Part $(i)$ is proved in Corollary 2.4. It was already known when $F=\mathbb{Q}$. In fact, if $F=\mathbb{Q}$, it was shown in [RSY, Theorem 3.1] that either

$$
\mathfrak{D}=(m) \text { or }(m N) \text { for some prime } N \text {. }
$$

The proof of this result relies on a careful study of the closed fibers of Morita's model of the Atkin-Lehner quotients of the Shimura curve of discriminant $\mathfrak{D}$; it is reasonable to expect that, under certain hypothesis, a similar result should hold for higher degree number fields $F$. See the discussion following Corollary 3.8 for an approach to this question by a different method.

In Table 1 we quote the existence of a modular abelian variety $A / \mathbb{Q}$ of dimension 4 and level 3872 such that $\operatorname{End}_{\mathbb{Q}}^{0}(A)=F(\sqrt{m})$, where $m \in F=\mathbb{Q}(\sqrt{5}), \mathrm{N}_{F / \mathbb{Q}}(m)=55$, and $\mathcal{O}=\operatorname{End}_{\mathbb{Q}(\sqrt{-1})}(A)$ is an order in the quaternion algebra $B$ over $F$ of discriminant $\mathfrak{D}=2 \cdot(4+\sqrt{5})$. By $(i), \mathcal{O}$ is not maximal in $B$.

Part (ii) is proved in Corollary 3.7 and (iii) in Theorem 4.1.

As for (iii), note firstly that for a given fixed $g$, the condition on $F$ not to contain $\mathbb{Q}\left(\zeta_{n}+\zeta_{n}^{-1}\right)$ for any $n \neq 1,2,3,4,6$ excludes only finitely many fields $F$. For instance, 
when $g=2$ this condition is empty, whereas for $g=4$ this only excludes $F=\mathbb{Q}(\sqrt{2})$ and $\mathbb{Q}(\sqrt{5})$.

In fact, the restriction on $F$ can be relaxed (by allowing $\zeta_{n}+\zeta_{n}^{-1} \in F$ for larger values of $n$ ) at the cost of additional items in part (iii). This can be achieved by following the proof of Theorem 4.1; we leave the details to the interested reader.

The condition on $\ell$ not to be inert in $K$ can not be removed; as for the requirement that $\sqrt{\ell}, \sqrt{2 \ell}, \sqrt{3 \ell}, \sqrt{2 \ell \pm \sqrt{3} \ell} \notin F$, see Theorem 4.1 for a statement without this hypothesis.

Roughly, (iii) claims that for half of the primes $\ell$, we have $\left(\frac{-\ell}{\mathfrak{N}}\right)=-1$ for all $\mathfrak{N} \mid \mathfrak{D}$ unless all $\mathfrak{N} \mid \frac{\mathfrak{D}}{(\mathfrak{D}, 2 m)}$ lie in the exceptional set $\mathcal{N}_{\ell}$. Here, by half of the primes we mean those $\ell$ such that $\left(\frac{K}{\ell}\right) \neq-1$ and $\sqrt{\ell}, \sqrt{2 \ell}, \sqrt{3 \ell}, \sqrt{2 \ell \pm \sqrt{3} \ell} \notin F$.

When $F=\mathbb{Q}$ and $\mathfrak{D}=(m N)$ for an odd prime $N$, (iii) -for $\ell=2,3$, use the slightly stronger Theorem 4.1- says that for any $\ell$ such that $\left(\frac{K}{\ell}\right) \neq-1$, either

- $N \in \mathcal{N}_{\ell}$, or

- For all primes $p \mid \mathfrak{D}:\left(\frac{-\ell}{p}\right) \neq 1$ if $\ell \neq 2($ resp. $p \not \equiv 1 \bmod 8$ if $\ell=2)$.

When trying to apply $($ iii $)$ to preclude explicit families of pairs $(\mathcal{O}, R)$ from being modular, it naturally arises the question of how does $K$ depend on $(\mathcal{O}, R)$. By means of the theory of descent applied to certain unramified covers of Shimura varieties, we prove in $[$ Ro] the following fact. See $[\mathrm{RSY}, \S 5]$ for a particular case with $F=\mathbb{Q}$.

Proposition 1.3. Let $\left(\mathcal{O}_{\mathfrak{D}}, R_{F(\sqrt{m})}, K\right)$ be a modular triplet. Assume that

- $m R_{F}$ is a square-free ideal, with $m=3$ or $\tau(m)>4$ for some embedding $\tau: F \hookrightarrow \mathbb{R}$, and

- There exists $\mathfrak{N} \mid \mathfrak{D}, \mathfrak{N} \nmid 2 m$, of odd residual degree $f(\mathfrak{N} / N)$.

Then $K$ is unramified away from $\mathrm{N}_{F / \mathbb{Q}}(\mathfrak{D}) \cdot \operatorname{disc}(F / \mathbb{Q})$.

The virtue of the above result is that, when the hypothesis is satisfied, there is a finite number of possibilities for $K$. Observe that, when $F=\mathbb{Q}$, the hypothesis of Proposition 1.3 simply require that $\mathfrak{D}=(m N)$ with $m, N>2$.

In addition, notice that the isomorphism (1) $B \simeq\left(\frac{-d, m}{F}\right)$ and Corollary 3.8 impose further restrictions on $K$. Let us illustrate how the combination of these results can be used to prevent triplets $(\mathcal{O}, R, K)$ from being modular.

Theorem 1.4. Let $F=\mathbb{Q}$. Let $M, N$ be two different odd primes and assume $\left(\mathcal{O}_{M N}, R_{\mathbb{Q}(\sqrt{M})}\right)$ is a modular pair. Then $N \equiv 3 \bmod 4$ and $\left(\frac{-N}{M}\right)=-1$. Moreover,

(i) If $M \equiv 3 \bmod 4$, then $K=\mathbb{Q}(\sqrt{-N})$ and $\left(\frac{-\ell}{M}\right)=-1$ for any odd prime $\ell$ such that $\left(\frac{\ell}{N}\right)=1$ and $N \notin \mathcal{N}_{\ell}$.

(ii) If $M \equiv 1 \bmod 4$, then $K=\mathbb{Q}(\sqrt{-d})$ with $d=N$ or $M N$. If $d=N$, then $\left(\frac{-\ell}{M}\right)=-1$ for any $\ell$ such that $\left(\frac{\ell}{N}\right)=1$ and $N \notin \mathcal{N}_{\ell}$. If $d=M N$, then $N \equiv 3$ $\bmod 8$ and $N \in \mathcal{N}_{\ell}$ for all odd primes $\ell$ such that $\left(\frac{-M N}{\ell}\right)=1$.

This is proved in Section 4 . Since the finite sets $\mathcal{N}_{\ell}$ are easily computable, notice that, by taking explicit values of $\ell$, the above criterion precludes infinitely many pairs $\left(\mathcal{O}_{M N}, R_{\mathbb{Q}(\sqrt{M})}\right)$ from being modular. Similar examples with higher degree number fields $F$ can easily be worked out in the same way. 
The material of this note can be used to prove the non-existence of rational points (or at least, non-trivial rational points) on Atkin-Lehner quotients of Shimura varieties associated with totally indefinite quaternion algebras over totally real number fields. Care must be taken though because rational points over a field $K$ on these varieties do not quite correspond to abelian varieties (with extra structure) defined over $K$, but rather to those with $K$ as field of moduli; details will appear in [Ro].

See also [PY] for (non-overlapping) results in this direction in dimension 1, where it should be noticed that the methods used there in are completely different.

Acknowledgement. It is a pleasure to thank B. Jordan, J. Gonzalez, D. Lorenzini, J. Quer and the referee for their helpful remarks.

\section{Quaternion multiplication over number fields}

Let $A / \mathbb{Q}$ be an abelian variety of (even) dimension $g$ of $\mathrm{GL}_{2}$-type over $\mathbb{Q}$ and let $R=\operatorname{End}_{\mathbb{Q}}(A) \subset E=\operatorname{End}_{\mathbb{Q}}(A) \otimes \mathbb{Q}$. Let $K$ be the minimal field over which all endomorphisms of $A \otimes \overline{\mathbb{Q}}$ are defined. Assume $\mathcal{O}=\operatorname{End}_{K}(A)$ is an order in a division totally indefinite quaternion algebra $B$ over a totally real number field $F$.

For any prime $N$, the action of $G_{\mathbb{Q}}$ on the Tate module $V_{N}(A)$ of $A$ induces a Galois representation

$$
\mathcal{R}_{N}: G_{\mathbb{Q}} \longrightarrow \mathrm{GL}_{2 g}\left(\mathbb{Q}_{N}\right),
$$

which in fact takes values in $\mathrm{GL}_{2}\left(R_{E}\right)$, independently of $\mathfrak{N}$.

For any prime ideal $\mathfrak{N}$ of $E$ over $N$, let $E_{\mathfrak{N}}$ stand for the completion of $E$ along $\mathfrak{N}$ and write $V_{\mathfrak{N}}(A)=V_{N}(A) \otimes_{\mathbb{Q}_{N}} E_{\mathfrak{N}}$. Since the representation $\mathcal{R}_{N}$ is compatible with the action of $E$, it induces a Galois representation

$$
r_{\mathfrak{N}}: G_{\mathbb{Q}} \longrightarrow \mathrm{GL}_{2}\left(E_{\mathfrak{N}}\right) .
$$

Lemma 2.1. Let $\ell$ be a prime number. There exists a finite extension $L_{\ell} / \mathbb{Q}_{\ell}$ such that the closed fibre of the Néron model of $A \otimes_{\mathbb{Q}} L_{\ell}$ over the ring of integers of $L_{\ell}$ is an abelian variety $\tilde{A}$ over $\mathbb{F}_{\ell}$.

Proof. By [Ri1, Theorem 3], $A$ has potential good reduction at $\ell$. Under this assumption, Serre and Tate explicitly construct $L_{\ell}$ and $\tilde{A} / \mathbb{F}_{\ell}$ at the end of p. 498 of $[\mathrm{SeTa}]$.

Remark 2.2. Let $A / \mathbb{Q}_{\ell}$ be an abelian variety with potential good reduction. By definition, there exist finite extensions $L / \mathbb{Q}_{\ell}$ over which $A$ acquires good reduction. Lemma above claims that $L$ can be chosen to have residue field $\mathbb{F}_{\ell}$. Note that in general there does not exist a minimal extension $L / \mathbb{Q}_{\ell}$ over which $A$ has good reduction (cf. [SeTa, p. 498] for a proof of this fact when the residue field is algebraically closed). Hence, there may exist good reduction extensions $L / \mathbb{Q}_{\ell}$ for $A$ with non trivial residual degree such that $A \times M$ has bad reduction for any subextension $M \varsubsetneqq L$.

Let $\ell$ be a prime and let $\tilde{A} / \mathbb{F}_{\ell}$ denote the closed fibre of the Néron model of $A \otimes L_{\ell}$ as in the lemma above.

Let $\operatorname{Fr}_{\ell} \in \operatorname{Gal}\left(\overline{\mathbb{F}}_{\ell} / \mathbb{F}_{\ell}\right)$ be the Frobenius automorphism. Let $\varphi_{\ell}$ be an element of $\operatorname{Gal}\left(\overline{\mathbb{Q}}_{\ell} / L_{\ell}\right)$ whose image in $\mathrm{Gal}\left(\overline{\mathbb{F}}_{\ell} / \mathbb{F}_{\ell}\right)$ under the canonical reduction map is $\mathrm{Fr}_{\ell}$. By Serre-Tate's criterion [SeTa, Thm. 1], $r_{\mathfrak{N}}\left(\varphi_{\ell}\right)$ is a well-defined element of $\mathrm{GL}_{2}\left(R_{E}\right)$ up to conjugation. 
Let $P_{\ell}(T):=P_{\varphi_{\ell}}(T) \in R_{E}[T]$ denote the characteristic polynomial of $r_{\mathfrak{N}}\left(\varphi_{\ell}\right)$. We shall write $a_{\ell}=\operatorname{Tr}\left(r_{\mathfrak{N}}\left(\varphi_{\ell}\right)\right) \in R_{E}$. By the work of Weil, $\left|\tau\left(a_{\ell}\right)\right| \leq 2 \sqrt{\ell}$ for any embedding $\tau: E \rightarrow \mathbb{C}$. By [Ri2, Prop. 3.5], $E=\mathbb{Q}\left(\left\{a_{\ell}\right\}_{\ell \nmid N_{A}}\right)$, where $N_{A}$ stands for the conductor of $A$.

There is a natural action of $G_{\mathbb{Q}}$ on the ring of endomorphisms $\mathcal{O}=\operatorname{End}_{\overline{\mathbb{Q}}}(A)$ of $A \otimes \overline{\mathbb{Q}}$. By the Skolem-Noether theorem, for any $\sigma \in G_{\mathbb{Q}}$ the automorphism $B \rightarrow B$, $\beta \mapsto \beta^{\sigma}$ is inner: there exists $\omega_{\sigma} \in \mathcal{O}$ such that $\beta^{\sigma}=\omega_{\sigma} \beta \omega_{\sigma}^{-1}$. Thus, $\omega_{\sigma}$ belongs to the normalizer $\operatorname{Norm}_{B}(\mathcal{O})$ of $\mathcal{O}$ in $B$, because $\beta^{\sigma} \in \mathcal{O}$ for any $\beta \in \mathcal{O}$.

Since the endomorphisms of $R \subset \mathcal{O}$ are defined over $\mathbb{Q}$, it follows that $\omega_{\sigma}$ belongs to the commutator of $E$ in $B$, which is $E$ itself because it is a maximal subfield of $B$. Hence, $\omega_{\sigma} \in R=E \cap \mathcal{O}$. This induces a continuous homomorphism

$$
\psi: G_{\mathbb{Q}} \longrightarrow E^{*} / F^{*}, \quad \sigma \mapsto \omega_{\sigma}
$$

Lemma 2.3. $\quad$ (i) $K$ is imaginary quadratic, say $K=\mathbb{Q}(\sqrt{-d}), d>0$.

(ii) $E=F(\omega)$ for an element $\omega \in \mathcal{O} \cap \operatorname{Norm}_{B}(\mathcal{O}), \omega^{2}=m$, where $m \in R_{F}$ is square-free and totally positive.

Proof. By $\left[\mathrm{Ri} 2\right.$, Lemma 3.1], $\operatorname{det}\left(r_{\mathfrak{N}}\right)=\epsilon \cdot \chi_{N}: G_{\mathbb{Q}} \longrightarrow E^{*}$, where $\chi_{N}$ is the $N$-adic cyclotomic character and $\epsilon: G_{\mathbb{Q}} \rightarrow E^{*}$ is a character of finite order.

Let us first show that $E$ is totally real. Indeed, if it were not, $E$ would be a CMfield. Let $\mathfrak{L}$ be the set of primes $\ell \nmid N_{A}$ such that $a_{\ell} \neq 0$. If $\epsilon\left(\varphi_{\ell}\right)=1$ then $a_{\ell} \in F^{*}$ by [Ri2, Proposition 3.4] and the fact that $E$ is a totally imaginary quadratic extension of $F$. Since $\psi\left(\varphi_{\ell}\right) \in a_{\ell} \cdot F^{*}$ by $\left[\mathrm{Ri} 2\right.$, Theorem 5.5], we deduce in turn that $\psi\left(\varphi_{\ell}\right) \in F^{*}$, thus $\varphi_{\ell} \in \operatorname{Ker}(\psi)$. Cebotarev Density Theorem together with the fact that $\mathfrak{L}$ has density 1 within the set of all primes (cf. [Se2]) would imply that $\operatorname{Ker}(\epsilon) \subseteq \operatorname{Ker}(\psi)$, whence $K \subseteq \overline{\mathbb{Q}}^{\operatorname{Ker}(\epsilon)}$. By [Ri2, Lemma 3.2], $\epsilon(c)=1$ for any complex conjugation $c \in G_{\mathbb{Q}}$ on $\overline{\mathbb{Q}}$ and we deduce that $K$ would be totally real. Theorem 2 in [Ri1] would apply to say that $B$ is not division, which contradicts our assumptions.

Since $E$ is totally real, it contains no roots of unity $\zeta \neq \pm 1$ and any non-trivial finite subgroup of $E^{*} / F^{*}$ has order 2. As $\psi$ embeds $\operatorname{Gal}(K / \mathbb{Q})$ in $E^{*} / F^{*}$, we obtain that $K$ is quadratic. Theorem 2 of [Ri1] implies that it is imaginary and $(i)$ follows.

Write $\operatorname{Gal}(K / \mathbb{Q})=\{1, \sigma\}$ and let $\omega \in R$ such that $\psi(\sigma) \in \omega \cdot F^{*}$. As we argued above, $\omega \in \mathcal{O} \cap \operatorname{Norm}_{B}(\mathcal{O})$. Since $\sigma^{2}=1, \omega^{2}=m \in R_{F}$ and we can assume $m$ is square-free. This shows $(i i)$.

Recall that an order $\mathcal{O}$ in $B$ is an Eichler order if it is the intersection of two maximal orders (cf. [Vi, p. 39, 84]). All orders with square-free discriminant $\operatorname{disc}(\mathcal{O})$ are Eichler orders by [Vi, p. 84 and p. 98, Ex. 5.3].

Corollary 2.4. Assume $\mathcal{O}$ is an Eichler order. Then $m \cdot R_{F}=\mathfrak{m}_{0}^{2} \cdot \mathfrak{m}$, where $\mathfrak{m}_{0}$ and $\mathfrak{m}$ are integral ideals of $F$ and $\mathfrak{m} \mid \operatorname{disc}(\mathcal{O})$. If the class number of $F$ is $h(F)=1$, then $m \mid \operatorname{disc}(\mathcal{O})$.

Proof. Let $\omega \in \mathcal{O} \cap \operatorname{Norm}_{B}(\mathcal{O})$ as in the above proof. By [Vi, p. 99, Ex. 5.4], $\omega$ normalizes $\mathcal{O}_{\wp}$ in $B_{\wp}$ for any prime ideal $\wp$ of $F$. By [Vi, p. 34 and 40], there are no restrictions on $m=\mathrm{n}(\omega)$ at any $\wp \mid \operatorname{disc}(\mathcal{O})$. By [Vi, p. 40], $m \cdot R_{F_{\wp}}$ is an even power of $\wp R_{F_{\wp}}$ for any $\wp \nmid \operatorname{disc}(\mathcal{O})$. This shows the first part. If $h(F)=1$, all ideals of $R_{F}$ are principal. Since $m$ is square-free, $\mathfrak{m}_{0}=(1)$. 
Since $E$ is totally real, the character $\epsilon$ mentioned in the proof above is trivial (cf. [Ri2, p. 244]) and [Ri2, Theorem 5.3] asserts that $F=\mathbb{Q}\left(\left\{a_{\ell}^{2}\right\}_{\ell \nmid N_{A}}\right)$. Also, by [Ri2, Lemma 3.1] $\operatorname{det}\left(r_{\mathfrak{N}}\left(\varphi_{\ell}\right)\right)=\ell$ and $P_{\ell}(T)=T^{2}-a_{\ell} T+\ell$ for any prime $\ell$.

For primes $\ell \nmid N_{A}$ such that $a_{\ell} \neq 0$ we have by [Ri2, Theorem 5.5] that if $\ell$ remains inert in $K$, then $a_{\ell} \in F^{*} \cdot \omega$. Otherwise, $a_{\ell} \in F^{*}$ and $P_{\ell} \in R_{F}[T]$.

In the terminology of modular forms, this is equivalent to saying that $A$ is the abelian variety attached (up to isogeny) to a normalized newform $f=\sum a_{n} q^{n} \in$ $S_{2}\left(\Gamma_{0}(L)\right)$ with an extra-twist by $\psi$. As we have seen, $F=\mathbb{Q}\left(\left\{a_{\ell}^{2}\right\}\right), E=F(\sqrt{m})=$ $\mathbb{Q}\left(\left\{a_{\ell}\right\}\right), K=\mathbb{Q}(\sqrt{-d})=\operatorname{Ker} \psi$ and $B=\left(\frac{-d, m}{F}\right)$ are completely determined by the coefficients $a_{\ell}$. With this perspective in mind, the conjecture addressed in this note claims that the Fourier coefficients of a normalized newform for $\Gamma_{0}(L), L \geq 1$, generate only finitely many endomorphism algebras $E, B$ of given degree over $\mathbb{Q}$.

\section{The case $\mathfrak{N}$ inert in $E$}

Keep the notation of Section 2. In addition, for the rest of the article we shall assume the following

Assumption. There exists a prime ideal $\mathfrak{N}_{0} \mid \operatorname{disc}(B), \mathfrak{N}_{0} \nmid 2 m$, at which $\mathcal{O}$ is locally maximal.

Fix such a prime $\mathfrak{N}_{0}$ and let $R_{0}=R \cap F$, a suborder of the ring of integers $R_{F}$ of $F$. By saying that $\mathcal{O}$ is locally maximal at $\mathfrak{N}_{0}$ we mean that $R_{0, \mathfrak{N}_{0}}$, the localization of $R_{0}$ at $\mathfrak{N}_{0}$, is the ring of integers of $F_{\mathfrak{N}_{0}}$ and that $\mathcal{O}_{\mathfrak{N}_{0}}$ is the (single) maximal order of $B_{\mathfrak{N}_{0}}$. Let $k_{0}$ be the residue field of $R_{0}$ at $\mathfrak{N}_{0}$, say of $\nu:=N^{f}$ elements for $f=f_{\mathfrak{N}_{0} / N} \geq 1$.

Having this, the condition on $\mathfrak{N}_{0}$ not to ramify in $E$ actually implies that $\mathfrak{N}_{0}$ remains inert in $R$. This follows from the fact that $R$ embeds optimally in $\mathcal{O}$, as by construction $R=E \cap \mathcal{O}$ (cf. [Vi, Ch. II, §3] for details on Eichler's theory of optimal embeddings). Let $k$ be the residue field of $\mathfrak{N}=\mathfrak{N}_{0} \cdot R$, which is a quadratic extension of $k_{0}$. Reducing mod $\mathfrak{N}$ we obtain the residual representation

$$
\bar{r}_{\mathfrak{N}}: G_{\mathbb{Q}} \longrightarrow \mathrm{GL}_{2}(k),
$$

that is, the representation of $G_{\mathbb{Q}}$ on $A[\mathfrak{N}]=\{x \in A: \beta \cdot x=0 \quad \forall \beta \in \mathfrak{N}\}$ of $A$.

Let $\pi_{0}$ be an uniformizer of $F_{\mathfrak{N}_{0}}$. By [Vi, Ch. II, $\S 1$ ], locally at $\mathfrak{N}_{0}$ the division quaternion algebra $B_{\mathfrak{N}_{0}}$ can be described as $B_{\mathfrak{N}_{0}}=E_{\mathfrak{N}}+E_{\mathfrak{N}} \pi$, where $\pi^{2}=\pi_{0}$ and $\beta \cdot \pi=\pi \cdot \beta^{\tau}$ for any $\beta \in E_{\mathfrak{N}}$. Here, $\tau$ denotes the non-trivial automorphism of $\operatorname{Gal}\left(E_{\mathfrak{N}} / F_{\mathfrak{N}_{0}}\right)$. Moreover, $\mathcal{O}_{\mathfrak{N}_{0}}=R_{\mathfrak{N}}+\cdot R_{\mathfrak{N}} \cdot \pi$.

Let $I=\left\{\beta \in \mathcal{O}: \mathrm{n}(\beta) \in \mathfrak{N}_{0}\right\}$. This is an ideal of $\mathcal{O}$ which locally at $\mathfrak{N}_{0}$ is $I_{\mathfrak{N}_{0}}=\mathfrak{N} \cdot R_{\mathfrak{N}}+R_{\mathfrak{N}} \cdot \pi$, whereas $I_{\mathfrak{M}_{0}}=\mathcal{O}_{\mathfrak{M}_{0}}$ at the remaining primes $\mathfrak{M}_{0} \neq \mathfrak{N}_{0}$ of $F$. Let $C=\operatorname{Ker}(I: A \rightarrow A)=\bigcap_{\beta \in I} \operatorname{Ker}(\beta: A \rightarrow A)$, a subgroup of $A[\mathfrak{N}]$.

The action of $B$ on $A(\overline{\mathbb{Q}})$ makes $V_{\mathfrak{N}}(A)$ a $B_{\mathfrak{N}_{0}}$-module, which must be free because $B_{\mathfrak{N}_{0}}$ is simple. In fact, since $\operatorname{dim}_{F_{\mathfrak{N}_{0}}}\left(B_{\mathfrak{N}_{0}}\right)=\operatorname{dim}\left(V_{\mathfrak{N}}(A)\right)=4$, we have $V_{\mathfrak{N}}(A) \simeq B_{\mathfrak{N}_{0}}$.

In the same fashion, $T_{\mathfrak{N}}(A)$ is naturally a module over $\mathcal{O}_{\mathfrak{N}_{0}}$. In fact, one can identify $T_{\mathfrak{N}}(A)$ as a left ideal of $\mathcal{O}_{\mathfrak{N}_{0}}$ by choosing an isomorphism $V_{\mathfrak{N}}(A) \simeq B_{\mathfrak{N}_{0}}$.

Since $\mathcal{O}_{\mathfrak{N}_{0}}$ is a maximal order, all its ideals are principal by [Vi, p. 34] and we may write $T_{\mathfrak{N}}(A)=\mathcal{O}_{\mathfrak{N}_{0}} \cdot x$ for some $x \in A[\mathfrak{N}]$.

Note that $A[\mathfrak{N}]=T_{\mathfrak{N}}(A) / \mathfrak{N} \cdot T_{\mathfrak{N}}(A) \simeq \mathcal{O}_{\mathfrak{N}_{0}} / \mathfrak{N} \cdot \mathcal{O}_{\mathfrak{N}_{0}}$. Also, we have $C=\mathcal{O}_{\mathfrak{N}_{0}} / I_{\mathfrak{N}} \simeq$ $R_{\mathfrak{N}} / \mathfrak{N} \cdot R_{\mathfrak{N}} \simeq k$. As a $R$-module, $\operatorname{Aut}_{R}(C) \simeq k^{*}$. 
The action of $G_{\mathbb{Q}}$ on $\mathcal{O}$ leaves $I$ invariant because it is the single two-sided ideal of $\mathcal{O}$ of reduced norm $\mathfrak{N}_{0}$. Since $R=\operatorname{End}_{\mathbb{Q}}(A), G_{\mathbb{Q}}$ acts $R$-linearly on $C$. It thus induces a Galois representation

$$
\alpha_{\mathfrak{N}}: G_{\mathbb{Q}} \longrightarrow \operatorname{Aut}_{R}(C)=k^{*} .
$$

As in [Jo], the character $\alpha_{\mathfrak{N}}$ shall play a key role in what follows, since it encodes many of the arithmetic properties of the Galois representation of $G_{\mathbb{Q}}$ on the torsion of $A$. Recall that $\nu=\sharp k_{0}$ and that $\psi$ is a character of $G_{\mathbb{Q}}$ with values in $\{ \pm 1\}$.

Lemma 3.1. There exists a $k$-basis of $A[\mathfrak{N}]$ with respect to which

$$
\begin{aligned}
& \bar{r}_{\mathfrak{N}}: G_{\mathbb{Q}} \longrightarrow \mathrm{GL}_{2}(k) \\
& \sigma \quad \rightarrow \quad\left(\begin{array}{cc}
\psi(\sigma) \alpha_{\mathfrak{N}}(\sigma)^{\nu} & 0 \\
\beta_{\sigma} & \alpha_{\mathfrak{N}}(\sigma)
\end{array}\right)
\end{aligned}
$$

for some $\beta_{\sigma} \in k$.

Proof. Write $\mathcal{O}_{\mathfrak{N}_{0}}=R_{\mathfrak{N}}+R_{\mathfrak{N}} \cdot \pi$ as above and let $x \in A[\mathfrak{N}]$ such that $A[\mathfrak{N}]=$ $\mathcal{O}_{\mathfrak{N}_{0}} / \mathfrak{N O} \mathcal{N}_{\mathfrak{N}_{0}} \cdot x=R_{\mathfrak{N}} / \mathfrak{N} R_{\mathfrak{N}} \cdot x+R_{\mathfrak{N}} / \mathfrak{N} R_{\mathfrak{N}} \cdot \pi(x)$. We shall compute $r_{\mathfrak{N}}(\sigma)$ for $\sigma \in G_{\mathbb{Q}}$ with respect to the $k$-basis $\{x, \pi(x)\}$ of $A[\mathfrak{N}]$.

Let $\sigma \in G_{K}$. Since all endomorphisms of $A$ are defined over $K$ it follows that $\pi^{\sigma}=\pi$. Let $x^{\sigma}=\alpha_{\sigma} \cdot x+\beta_{\sigma} \cdot \pi(x)$ for some $\alpha_{\sigma}, \beta_{\sigma} \in R_{\mathfrak{N}}$. Then $(\pi(x))^{\sigma}=\pi^{\sigma}\left(x^{\sigma}\right)=$ $\pi\left(\alpha_{\sigma} \cdot x+\beta_{\sigma} \cdot \pi(x)\right)=\alpha_{\sigma}^{\tau} \pi(x)+\beta_{\sigma}^{\tau} \pi^{2}(x)=\alpha_{\sigma}^{\tau} \pi(x)$, because $\pi^{2}=\pi_{0}$ and $\pi_{0} \cdot x=0$. Switching $\alpha_{\sigma}$ by $\alpha_{\sigma}^{\tau}$ and reducing $\bmod \mathfrak{N}$ it follows that $r_{\mathfrak{N}}(\sigma)=\left(\begin{array}{cc}\alpha_{\sigma}^{\nu} & 0 \\ \beta_{\sigma} & \alpha_{\sigma}\end{array}\right)$. In particular we recover the canonical character $\alpha_{\mathfrak{N}}: G_{\mathbb{Q}} \rightarrow k^{*}$ as $\alpha_{\mathfrak{N}}(\sigma)=\alpha_{\sigma}$.

Let $c \in G_{\mathbb{Q}}$ denote a complex conjugation. As in Lemma 2.3 and its proof, for all $\beta \in \mathcal{O}$ we have $\beta^{c}=\omega \beta \omega^{-1}, \omega \in R, \omega^{2}=m \in F^{*}$.

It follows that $\pi^{c}=\omega \pi \omega^{-1}=\pi \omega^{\tau} \omega^{-1}=-\pi$. Write $x^{c}=\alpha_{c} x+\beta_{c} \pi(x)$ for some $\alpha_{c}, \beta_{c} \in R_{\mathfrak{N}}$. Then $(\pi(x))^{c}=\pi^{c}\left(x^{c}\right)=-\pi\left(\alpha_{c} x+\beta_{c} \pi(x)\right)=-\pi_{0} \beta_{c}^{\tau} x-$ $\alpha_{c}^{\tau} \pi(x)=-\alpha_{c}^{\tau} \pi(x)$. In particular $\alpha_{\mathfrak{N}}(c)=-\alpha_{c}^{\tau}$. Since $c^{2}=\operatorname{Id}, \alpha_{c}^{\tau}= \pm 1$ and $\bar{r}_{\mathfrak{N}}(c)=\left(\begin{array}{cc} \pm 1 & 0 \\ \beta_{c} & \mp 1\end{array}\right)$. Either value takes $\bar{r}_{\mathfrak{N}}(c)$, the lemma follows.

Let $G_{\mathbb{Q}}^{a b}=\operatorname{Gal}\left(\mathbb{Q}^{a b} / \mathbb{Q}\right)$ denote the Galois group of the maximal abelian extension $\mathbb{Q}^{a b}$ of $\mathbb{Q}$. For every prime $\ell$, let $\mathbb{Q}_{\ell}^{a b}$ denote the maximal abelian extension of $\mathbb{Q}_{\ell}$ and let $G_{\ell}^{a b}=\operatorname{Gal}\left(\mathbb{Q}_{\ell}^{a b} / \mathbb{Q}_{\ell}\right)$. Let $I_{\ell}^{a b}$ denote the inertia subgroup of $G_{\ell}^{a b}$, which we regard as a subgroup of $G_{\mathbb{Q}}^{a b}$. Local class field theory establishes a canonical isomorphism

$$
\omega_{\ell}: \mathbb{Z}_{\ell}^{*} \stackrel{\sim}{\longrightarrow} I_{\ell}^{a b}
$$

the local Artin reciprocity map. Global class field theory yields a surjective map

$$
\omega: \prod_{\ell} \mathbb{Z}_{\ell}^{*} \stackrel{\prod \omega_{\ell}}{\rightarrow} G_{\mathbb{Q}}^{a b} .
$$

We shall denote

$$
\varrho_{\ell}: \mathbb{Z}_{\ell}^{*} \stackrel{\omega_{\ell}}{\simeq} I_{\ell}^{a b} \subset G_{\mathbb{Q}}^{a b} \stackrel{\alpha_{\mathfrak{N}}}{\longrightarrow} k^{*}
$$

the composition of the local Artin reciprocity map together with the character $\alpha_{\mathfrak{N}}$ acting on the canonical torsion subgroup $C$. In particular, since $\varrho_{N}: \mathbb{Z}_{N}^{*} \longrightarrow k^{*}$ is a continuous homomorphism, $\varrho_{N}\left(\mathbb{Z}_{N}^{*}\right) \subseteq \mathbb{F}_{N}^{*} \subset k^{*}$. 
Definition 3.2. Let $\kappa(A, \mathfrak{N})$ be the least common multiple of 4 and the orders of $\alpha_{\mathfrak{N}}\left(I_{\ell}\right)$ as $\ell$ runs over all prime integers $\ell \neq N$.

Since $A$ has good reduction outside a finite set of primes, and potential good reduction at those, $\kappa(A, \mathfrak{N})$ is a well-defined integer.

For a prime $\ell$, let $\mathfrak{a}_{\ell}=\prod \mathfrak{L}$, where $\mathfrak{L}$ runs over the prime ideals of $F$ above $\ell$ such that $\left[F_{\mathfrak{L}}: \mathbb{Q}_{\ell}\right]$ is odd. Let $B(\ell)$ be the totally definite quaternion algebra over $F$ of discriminant $\frac{\mathfrak{D} \cdot \mathfrak{a}_{\ell}}{\left(\mathfrak{D}, \mathfrak{a}_{\ell}\right)^{2}}$.

Definition 3.3. Let $\kappa(B)$ be the least common multiple of 2 and the positive integers $n \geq 3$ such that

(i) $\mathbb{Q}\left(\zeta_{n}+\zeta_{n}^{-1}\right) \subseteq F$, and

(ii) For all $\ell \mid \mathrm{N}_{F / \mathbb{Q}}(\mathfrak{D})$, no prime ideal $\wp \mid \frac{\mathfrak{D}}{\left(\mathfrak{D}, \mathfrak{a}_{\ell}\right)}$ splits in $F\left(\zeta_{n}\right)$.

For instance, when $F=\mathbb{Q}$ or $F$ quadratic, $F \neq \mathbb{Q}(\sqrt{2}), \mathbb{Q}(\sqrt{5})$, we have $\kappa(B) \mid 12$. If $F=\mathbb{Q}(\sqrt{2}), \kappa(B) \mid 24$. If $F=\mathbb{Q}(\sqrt{5}), \kappa(B) \mid 60$.

For arbitrary $F$, we have $\kappa(B) \mid \operatorname{lcm}_{\varphi(n) \mid g}(n)$, since $\left[\mathbb{Q}\left(\zeta_{n}\right): \mathbb{Q}\right]=\varphi(n)=\sharp(\mathbb{Z} / n \mathbb{Z})^{*}$. Also, $8 \nmid \kappa(B)$ provided $\sqrt{2} \notin F$, because $\mathbb{Q}\left(\zeta_{8}+\zeta_{8}^{-1}\right)=\mathbb{Q}(\sqrt{2})$.

Lemma 3.4. $\kappa(A, \mathfrak{N}) \mid 2 \cdot \kappa(B)$.

Proof. Let $\ell$ be a prime and $v$ a place of $K$ over $\ell$. Since $\left[I_{\ell}: I_{v}\right] \leq 2$, it suffices to show that $\alpha_{\mathfrak{N}}^{\kappa(B)}\left(I_{v}\right)=\{1\}$ for all places $v \nmid N$ of $K$.

Let $L_{\ell} / \mathbb{Q}_{\ell}$ be as in Lemma 2.1 the extension constructed by Serre and Tate over which $A$ acquires good reduction. Let $\tilde{A} / \mathbb{F}_{\ell}$ denote the closed fibre of the Néron model $\mathcal{A}$ of the abelian variety $A \otimes L_{\ell}$.

Let $\overline{\mathbb{F}}_{\ell}$ be a fixed algebraic closure of $\mathbb{F}_{\ell}$. Let $\tilde{B}=\operatorname{End}_{\overline{\mathbb{F}}_{\ell}}(\tilde{A}) \otimes \mathbb{Q}$ be the algebra of endomorphisms of $\tilde{A} \otimes \overline{\mathbb{F}}_{\ell}$, in which $B$ is embedded.

The group $I_{v}$ acts on $\mathcal{A} \times K_{v}$ via the Galois action on $L_{\ell} \cdot K_{v}$ over $K_{v}$. Since $B=\operatorname{End}_{K}^{0}(A)$, the action of $I_{v}$ commutes with that of $B$.

In addition, $I_{v}$ acts trivially on the residue field of $L_{\ell} \cdot K_{v}$ and thus specializes to an action on the closed fibre of $\mathcal{A}$ by algebraic automorphisms (cf. [SeTa, p. 497]). By considering the action both of $I_{v}$ and $\tilde{B}$ on $V_{\mathfrak{N}}\left(A \times L_{\ell} \cdot K_{v}\right)=V_{\mathfrak{N}}(\tilde{A})$ we obtain that $r_{\mathfrak{N}}\left(I_{v}\right)$ is a finite subgroup of the group $G$ of units of an order in $C_{\tilde{B}}(B)$, the centralizer of $B$ in $\tilde{B}$.

As in the proof of Proposition 5.2 in $[\mathrm{Mi}], C_{\tilde{B}}(B)$ is either $(i)$ a totally imaginary quadratic extension of $F$ which splits $B$ or $(i i) B(\ell)$.

In $(i), A$ is isogenous over $\overline{\mathbb{F}}_{\ell}$ to $A_{0}^{2}$, where $A_{0} / \overline{\mathbb{F}}_{\ell}$ is an abelian variety of dimension $g / 2$ with $\operatorname{End}_{\overline{\mathbb{F}}_{\ell}}\left(A_{0}\right) \supseteq C_{\tilde{B}}(B)$. In $(i i) A / \overline{\mathbb{F}}_{\ell}$ is supersingular: it is isogenous to $g$ copies of a supersingular elliptic curve.

If $(i)$ holds, $G$ is the cyclic group of $n$-th roots of unity in the CM-field $C_{\tilde{B}}(B)$ for some $n \geq 1$. As soon as $n>2, C_{\tilde{B}}(B)=F\left(\zeta_{n}\right)$. Since it splits $B$, no prime $\wp \mid \mathfrak{D}$ splits in $F\left(\zeta_{n}\right)$.

Assume now that (ii) holds. If $G \supseteq\left\langle\zeta_{n}\right\rangle$ for some $n>2$, then $F\left(\zeta_{n}\right)$ is a quadratic extension of $F$ which embeds in $B(\ell)$. We again deduce that no $\wp \mid \operatorname{disc}(B(\ell))$ splits in $F\left(\zeta_{n}\right)$. If $\ell \nmid \mathrm{N}_{F / \mathbb{Q}}(\mathfrak{D})$, this implies that no $\wp \mid \mathfrak{D} \cdot \mathfrak{a}_{\ell}$ splits in $F\left(\zeta_{n}\right)$. If $\ell \mid \mathrm{N}_{F / \mathbb{Q}}(\mathfrak{D})$, we obtain that no $\wp \mid \frac{\mathfrak{D}}{\left(\mathfrak{D}, \mathfrak{a}_{\ell}\right)}$ splits in $F\left(\zeta_{n}\right)$. 
By Lemma 3.1, there exists a surjective homomorphism from $r_{\mathfrak{N}}\left(I_{v}\right)$ onto $\alpha_{\mathfrak{N}}\left(I_{v}\right)$. Since $\alpha_{\mathfrak{N}}\left(I_{v}\right) \subseteq k^{*}$, this is a cyclic group. The discussion above yields that $\alpha_{\mathfrak{N}}\left(I_{v}\right)^{n}=$ $\{1\}$ for some $n$ as in the statement.

The above proof shows a slightly stronger fact: If $\ell \nmid \mathrm{N}_{F / \mathbb{Q}}(\mathfrak{D})$ is a supersingular prime for $A$, then no $\wp \mid \mathfrak{D} \cdot \mathfrak{a}_{\ell}$ splits in $F\left(\zeta_{n}\right)$. We did not include this in the statement of Lemma 3.4 because we shall apply it to any modular abelian variety $A / \mathbb{Q}$ with multiplication by $B$. One can not expect to find a prime $\ell \nmid \mathrm{N}_{F / \mathbb{Q}}(\mathfrak{D})$ which is supersingular for all such $A$ simultaneously.

Corollary 3.5. Assume $K=\mathbb{Q}(\sqrt{-N})$ and $4 \mid \kappa(B)$. Then $\kappa(A, \mathfrak{N}) \mid \kappa(B)$.

Proof. In the above proof we showed that $\alpha_{\mathfrak{N}}^{\kappa(B)}\left(I_{v}\right)=\{1\}$ for all places $v \nmid N$ of $K$. Since $K=\mathbb{Q}(\sqrt{-N})$, we have $I_{v}=I_{\ell}$ for all these places of $K$. As $4 \mid \kappa(B)$, it follows from the definition of $\kappa(A, \mathfrak{N})$ that it divides $\kappa(B)$.

Proposition 3.6. For any prime $\ell \neq N, \alpha_{\mathfrak{N}}\left(\varphi_{\ell}^{\kappa(A, \mathfrak{N})}\right)=\ell^{\kappa(A, \mathfrak{N}) / 2}$.

Proof. Let $\kappa=\kappa(A, \mathfrak{N})$. Let us first show that $\alpha_{\mathfrak{N}}\left(\varphi_{\ell}^{\kappa}\right)=\varrho_{N}^{\kappa}\left(\frac{1}{\ell}\right) \in \mathbb{F}_{N}^{*}$. The image by $\omega$ of the tuple

$$
\left(\frac{1}{\ell}, \ldots, \frac{1}{\ell}, \stackrel{\ell}{1}, \frac{1}{\ell}, \ldots, \frac{1}{\ell}\right) \in \prod_{p} \mathbb{Z}_{p}^{*}
$$

is an element $\tilde{\varphi}_{\ell} \in G_{\mathbb{Q}}^{a b}$ which reduces to the Frobenius automorphism $\operatorname{Fr}_{\ell} \in$ $\operatorname{Gal}\left(\overline{\mathbb{F}}_{\ell} / \mathbb{F}_{\ell}\right)$. Hence $\varphi_{\ell} \circ \tilde{\varphi}_{\ell}^{-1} \in I_{\ell}$ and $\alpha_{\mathfrak{N}}\left(\varphi_{\ell}^{\kappa}\right)=\alpha_{\mathfrak{N}}\left(\tilde{\varphi}_{\ell}^{\kappa}\right)$ because $\alpha_{\mathfrak{N}}^{\kappa}\left(I_{\ell}\right)=\{1\}$. It follows that $\alpha_{\mathfrak{N}}^{\kappa}\left(\varphi_{\ell}\right)=\varrho_{\ell}^{\kappa}(1) \cdot \varrho_{N}^{\kappa}\left(\frac{1}{\ell}\right) \cdot \prod_{p \neq N, \ell} \varrho_{p}^{\kappa}\left(\frac{1}{\ell}\right)=\varrho_{N}^{\kappa}\left(\frac{1}{\ell}\right)$.

Let $\bar{\chi}_{N}: G_{\mathbb{Q}} \longrightarrow \mathbb{F}_{N}^{*}$ be the reduction of the cyclotomic character $\bmod N$. By $[\operatorname{Se} 1$, Prop. 3, 8] we have $\bar{\chi}_{N \mid I_{N}^{a b}} \cdot \omega_{N}(x)=\frac{1}{x} \bmod N$ for all $x \in \mathbb{Z}_{N}^{*}$.

Since $\mathbb{F}_{N}^{*}$ has order $N-1$, we have

$$
\varrho_{N}(x)=x^{-c} \bmod N \text { for some } 0 \leq c<N-1 .
$$

By Lemma 3.1, since $\operatorname{det}\left(r_{\mathfrak{N}}\right)=\chi_{N}$ we have $\frac{1}{x}=\bar{\chi}_{N}\left(\omega_{N}(x)\right)=\operatorname{det} \bar{r}_{\mathfrak{N}}\left(\omega_{N}(x)\right)=$ $\psi\left(\omega_{N}(x)\right) \cdot N_{k / k_{0}}\left(\alpha_{\mathfrak{N}}\left(\omega_{N}(x)\right)=\psi\left(\omega_{N}(x)\right) N_{k / k_{0}}\left(\varrho_{N}(x)\right)\right.$.

Since $\varrho_{N}(x) \in \mathbb{F}_{N}^{*}, N_{k / k_{0}}\left(\varrho_{N}(x)\right)=\varrho_{N}^{2}(x)$ and it follows that $\varrho_{N}^{2}(x)=x^{-2 c}=$ $\pm x^{-1}$. Hence $2 c \equiv 1$ or $\frac{N+1}{2} \bmod N-1$. Since $N-1$ is even, we deduce that $N \equiv 3$ $\bmod 4$ and $c=\frac{N+1}{4}$ or $\frac{3 N-1}{4} \bmod N-1$. Thus $\varrho_{N}^{\kappa}(x) \equiv x^{-\frac{(N+1) \kappa}{4}}$ or $x^{-\frac{(3 N-1) \kappa}{4}} \bmod$ $N$. Since $4 \mid \kappa, \frac{(N+1) \kappa}{4} \equiv \frac{(3 N-1) \kappa}{4} \equiv \kappa / 2 \bmod N-1$ and $\alpha_{\mathfrak{N}}\left(\varphi_{\ell}^{\kappa}\right)=\ell^{\kappa / 2} \in \mathbb{F}_{N}^{*}$. Consequently, $\alpha_{\mathfrak{N}}\left(\varphi_{\ell}^{\kappa}\right)=\ell^{\kappa / 2}$.

During the proof of the above proposition we proved in passing the following.

Corollary 3.7. $N \equiv 3 \bmod 4$.

Corollary 3.8. If $\operatorname{ord}_{2}(\kappa(A, \mathfrak{N})) \leq \operatorname{ord}_{2}(\nu+1)$ then $K=\mathbb{Q}(\sqrt{-N})$.

Proof. Let $\kappa=\kappa(A, \mathfrak{N})$. For each $\ell \neq N$ we have $\ell \equiv \psi\left(\operatorname{Fr}_{\ell}\right) \alpha_{\mathfrak{N}}\left(\operatorname{Fr}_{\ell}\right)^{\nu+1} \bmod \mathfrak{N}$. Set $t=\kappa / 2^{\operatorname{ord}_{2}(\kappa)}$. Then $\kappa \mid t(\nu+1)$ and $\ell^{t} \equiv \psi^{t}\left(\operatorname{Fr}_{\ell}\right) \alpha_{\mathfrak{N}}^{t(\nu+1)}\left(\operatorname{Fr}_{\ell}\right)$. Since $t$ is odd and $\psi$ is a quadratic character, it follows from Proposition 3.6 that $\psi\left(\operatorname{Fr}_{\ell}\right) \equiv \ell^{t(\nu-1) / 2}$ 
$\bmod \mathfrak{N}$. As $\psi\left(\mathrm{Fr}_{\ell}\right), \ell \in \mathbb{Z}$ and $\mathfrak{N}=\mathfrak{N}_{0} \cdot R$, this is equivalent to the congruence $\psi\left(\operatorname{Fr}_{\ell}\right) \equiv \ell^{t(\nu-1) / 2} \bmod \mathfrak{N}_{0}$.

Since the residue field of $\mathfrak{N}_{0}$ is $k_{0}$, we obtain that $\psi\left(\operatorname{Fr}_{\ell}\right)=\left(\frac{\ell}{k_{0}}\right)$ for all $\ell \neq N$. If $f$ is even, then $\left(\frac{\ell}{k_{0}}\right)=1$ for all $\ell$ and $\psi$ is trivial, a contradiction. Hence $f$ is odd and $\left(\frac{\ell}{k_{0}}\right)=\left(\frac{\ell}{N}\right)=\left(\frac{-N}{\ell}\right)$. It follows that $\psi=\left(\frac{-N}{\cdot}\right)$ and $K=\mathbb{Q}(\sqrt{-N})$.

One application of Corollary 3.8 is in Theorem 1.4 (ii). It can also be used to show in many instances that an analogue of (3) in the Introduction holds for higher degree fields $F$.

Let for example $\left(\mathcal{O}_{\mathfrak{D}}, R_{F(\sqrt{m})}, K\right)$ be a modular triplet such that $\sqrt{2} \notin F$ and $m \mid \mathfrak{D}=\mathfrak{N}_{1} \cdot \ldots \cdot \mathfrak{N}_{2 r}$, above integer primes $N_{i}$. Order them in some way that $\mathfrak{N}_{i} \mid 2 m$ for $1 \leq i \leq s$ and $\mathfrak{N}_{i} \nmid 2 m$ for $i>s$. Assume $\mathfrak{N}_{2 r-1}$ and $\mathfrak{N}_{2 r}$ can be chosen such that $N_{2 r-1}, N_{2 r}$ are odd, $N_{2 r-1} \neq N_{2 r}$ and $f\left(\mathfrak{N}_{2 r} / N_{2 r}\right)$ is odd.

If either $(a) N_{i}, N_{j} \equiv 1 \bmod 4$ for some $1 \leq i \neq j \leq s$ or $(b) N_{2 r} \equiv 7 \bmod 8$, then $s \geq 2 r-1$.

Indeed, if $s \leq 2 r-2$, it would follow from (2) in the Introduction that $N_{2 r-1} N_{2 r}$ $\operatorname{disc}(K)$. By Definition 3.3, $4 \nmid \kappa(B)$ in $(a), 8 \nmid \kappa(B)$ in $(b)$. By Lemma 3.4, $8 \nmid$ $\kappa\left(A, \mathfrak{N}_{2 r}\right)$ in $(a), 16 \nmid \kappa\left(A, \mathfrak{N}_{2 r}\right)$ in $(b)$. Corollary 3.8 applied to $\mathfrak{N}_{2 r}$ would imply $K=\mathbb{Q}\left(\sqrt{-N_{2 r}}\right)$, a contradiction.

Lemma 3.9. The characteristic polynomial of $\varphi_{\ell}$ satisfies $P_{\ell}(T) \equiv T^{2}-\left(\alpha_{\mathfrak{N}}\left(\varphi_{\ell}\right)+\right.$ $\left.\ell \alpha_{\mathfrak{N}}\left(\varphi_{\ell}^{-1}\right)\right) \cdot T+\ell \in k[T]$.

Proof. It follows from Lemma 3.1 that for any $\sigma \in G_{\mathbb{Q}}$, the characteristic polynomial $P_{\sigma}(T) \in R[T]$ satisfies

$$
P_{\sigma} \bmod \mathfrak{N} \equiv T^{2}-\left(\alpha_{\mathfrak{N}}(\sigma)+\psi\left(\varphi_{\ell}\right) \alpha_{\mathfrak{N}}(\sigma)^{\nu}\right) \cdot T+\psi\left(\varphi_{\ell}\right) \operatorname{Nm}\left(\alpha_{\mathfrak{N}}(\sigma)\right) .
$$

Besides, as we mentioned at the end of Section 2, we have $P_{\ell}=P_{\varphi_{\ell}}=T^{2}-$ $a_{\ell} T+\ell$. Hence $\psi\left(\varphi_{\ell}\right) \alpha_{\mathfrak{N}}\left(\varphi_{\ell}\right)^{\nu} \alpha_{\mathfrak{N}}\left(\varphi_{\ell}\right)=\ell \in k$ and we deduce that $a_{\ell} \equiv \alpha_{\mathfrak{N}}\left(\varphi_{\ell}\right)+$ $\psi\left(\varphi_{\ell}\right) \alpha_{\mathfrak{N}}\left(\varphi_{\ell}\right)^{\nu} \equiv \alpha_{\mathfrak{N}}\left(\varphi_{\ell}\right)+\ell \alpha_{\mathfrak{N}}\left(\varphi_{\ell}^{-1}\right) \bmod \mathfrak{N}$.

\section{Proof of the main results}

Keep the notations of the previous section. Let $\lambda_{ \pm}=\sqrt{2 \ell \pm \sqrt{3}}$.

Theorem 4.1. Let $(\mathcal{O}, R, K)$ be a modular triplet. Assume $\zeta_{n}+\zeta_{n}^{-1} \notin F$ for any $n$-th primitive root of $1, n \neq 1,2,3,4,6$, and that $\mathcal{O}$ is locally maximal at some ideal $\mathfrak{N}_{0} \mid \operatorname{disc}(B), \mathfrak{N}_{0} \nmid 2 m$. Then, for any prime $\ell$ which splits or ramifies in $K$, either $\mathfrak{N}_{0} \in \mathcal{N}_{\ell}$ or

- $\left(\frac{-\ell}{\wp}\right) \neq 1$ for all $\wp \mid \mathfrak{D}$, or

- $\sqrt{2 \ell} \in F$ and $\left(\frac{-1}{\wp}\right) \neq 1$ for all $\wp \mid \mathfrak{D}, \wp \nmid \ell$, or

- $\sqrt{\ell}$ or $\sqrt{3 \ell} \in F$ and $\left(\frac{-3}{\wp}\right) \neq 1$ for all $\wp \mid \mathfrak{D}, \wp \nmid \ell$, or

- $\lambda_{ \pm} \sqrt{\ell} \in F$ and $\left(\frac{-1 \mp 4 \sqrt{3} / 7}{\wp}\right) \neq 1$ for all $\wp \mid \mathfrak{D}, \wp \nmid \ell$.

Proof. Let $A$ be a modular abelian variety over $\mathbb{Q}$ such that $\operatorname{End}_{\mathbb{Q}}(A)=R$ and $\operatorname{End}_{K}(A) \simeq \mathcal{O}$. 
By our assumption on $\mathfrak{N}_{0}$, it remains inert in $R$ and we let $\mathfrak{N}=\mathfrak{N}_{0} R$ (cf. Section 3 ). Let $\overline{\mathfrak{N}}$ be an ideal of $\overline{\mathbb{Q}}$ above $\mathfrak{N}_{0}$. Choose a prime $\ell$ such that $\mathfrak{N}_{0} \notin \mathcal{N}_{\ell}$ and $\left(\frac{K}{\ell}\right) \neq-1$. Then $\mathfrak{N}_{0} \nmid \ell, \varphi_{\ell} \in G_{K}$ and $a_{\ell} \in R_{F}$.

By Lemma 3.9, $\alpha_{\mathfrak{N}}\left(\varphi_{\ell}\right)+\ell \alpha_{\mathfrak{N}}\left(\varphi_{\ell}^{-1}\right)=\sqrt{\ell} \cdot\left(\frac{\alpha_{\mathfrak{N}}\left(\varphi_{\ell}\right)}{\sqrt{\ell}}+\left(\frac{\alpha_{\mathfrak{N}}\left(\varphi_{\ell}\right)}{\sqrt{\ell}}\right)^{-1}\right) \equiv a_{\ell} \bmod \overline{\mathfrak{N}}$. Here, $\zeta=\frac{\alpha_{\mathfrak{N}}\left(\varphi_{\ell}\right)}{\sqrt{\ell}}$ is a $\kappa(A, \mathfrak{N})$-th root of 1 by Proposition 3.6.

Since $\zeta_{n}+\zeta_{n}^{-1} \notin F$ for any $n \neq 1,2,3,4,6$, we have $\kappa(B) \mid 12$. Hence $\kappa(A, \mathfrak{N}) \mid 24$ by Lemma 3.4.

Computing the possible values of $\sqrt{\ell}\left(\zeta+\zeta^{-1}\right)$ for all 24-th roots of 1 , we obtain that $a_{\ell} \equiv 0, \sqrt{\ell}, \sqrt{2 \ell}, \sqrt{3 \ell}, 2 \sqrt{\ell}$ or $\lambda_{ \pm} \cdot \sqrt{\ell} \bmod \overline{\mathfrak{N}}$. In other words, $\mathfrak{N}_{0} \mid a_{\ell}^{2}-s \ell$ for some $s=0,1,2,3,4$ or $\mathfrak{N}_{0} \mid a_{\ell}^{4}-4 a_{\ell}^{2} \ell+\ell^{2}$.

As we already mentioned, $\left|\tau\left(a_{\ell}\right)\right| \leq 2 \sqrt{\ell}$ for any $\tau: F \hookrightarrow \mathbb{R}$. It follows from the very definition of $\mathcal{N}_{\ell}$ that the above congruence is in fact an identity: $a_{\ell}=$ $0, \sqrt{\ell}, \sqrt{2 \ell}, \sqrt{3 \ell}, 2 \sqrt{\ell}$ or $\lambda_{ \pm} \cdot \sqrt{\ell}$.

Since $\left(\frac{K}{\ell}\right) \neq-1$, the residue field of $K$ at $\ell$ is $\mathbb{F}_{\ell}$. By reducing the endomorphisms of $A \bmod \ell$ it follows that $B=\operatorname{End}_{K}(A) \otimes \mathbb{Q}$ embeds in $\operatorname{End}_{\mathbb{F}_{\ell}}(\tilde{A}) \otimes \mathbb{Q}$.

According to $[$ Ta1, Theorem 2 (a)], [Ta2, Theorem $1(1)], \operatorname{End}_{\mathbb{F}_{\ell}}(\tilde{A}) \otimes \mathbb{Q}$ is isomorphic to $\mathrm{M}_{2 r}(\tilde{F})$, where $\tilde{F}$ is the splitting field of $\prod_{\tau: F \hookrightarrow \mathbb{R}}\left(T^{2}-a_{\ell}^{\tau} T+\ell\right)$. Here, $r \mid g=\operatorname{dim}(A)$ and $[\tilde{F}: \mathbb{Q}]=g / r$.

From the above values for $a_{\ell}$ we have (in the same order) $\tilde{F}=\mathbb{Q}(\sqrt{-\ell}), \mathbb{Q}(\sqrt{\ell}$, $\sqrt{-3}), \mathbb{Q}(\sqrt{2 \ell}, \sqrt{-1}), \mathbb{Q}(\sqrt{3 \ell}, \sqrt{-3}), \mathbb{Q}(\sqrt{\ell})$ or $\mathbb{Q}\left(\lambda_{ \pm} \cdot \sqrt{\ell}, \sqrt{-1 \mp 4 \sqrt{3} / 7}\right)$.

Case $\tilde{F}=\mathbb{Q}(\sqrt{\ell})$ can not arise: we would have $\operatorname{End}_{\mathbb{F}_{\ell}}(A) \otimes \mathbb{Q} \simeq \mathrm{M}_{g}(\mathbb{Q}(\sqrt{\ell}))$ and this would say that $A$ is isogenous to $g$ copies of an elliptic curve $E / \mathbb{F}_{\ell}$ with $\operatorname{End}_{\mathbb{F}_{\ell}}(E) \otimes \mathbb{Q} \simeq \mathbb{Q}(\sqrt{\ell})$, which is not possible.

Let us show that $F \cdot \tilde{F}$ splits $B$ locally at all places $\wp \nmid \ell$, or equivalently, no prime $\wp \mid \mathfrak{D}, \wp \nmid \ell$, splits in the quadratic extension $F \cdot \tilde{F}$ of $F$.

The Tate module $V_{p}(\tilde{A})$ is a $F \cdot \tilde{F} \otimes \mathbb{Q}_{p}$-vector space of $\operatorname{rank} 2$. Since $B \subset \operatorname{End}_{\mathbb{F}_{\ell}}(\tilde{A}) \otimes$ $\mathbb{Q}$ and $\tilde{F}$ is the center of $\operatorname{End}_{\mathbb{F}_{\ell}}(\tilde{A}) \otimes \mathbb{Q}$, we conclude that $B$ acts $F \cdot \tilde{F} \otimes \mathbb{Q}_{p}$-linearly on $V_{p}(\tilde{A})$.

Thus $B \subset \mathrm{M}_{2}\left(F \cdot \tilde{F} \otimes \mathbb{Q}_{p}\right)$, which says that $F \cdot \tilde{F}$ splits $B$ locally at $\wp$.

Finally, when $\tilde{F}=\mathbb{Q}(\sqrt{-\ell})$, it is also clear that $F \cdot \tilde{F}=F(\sqrt{-\ell})$ also splits $B$ at the places $\wp \mid \ell$, because these prime ideals ramify in $F \cdot \tilde{F}$. This finishes the proof of the theorem.

Theorem 1.2 (iii) now follows automatically by applying Theorem 4.1 to each prime ideal $\mathfrak{N} \mid \mathfrak{D}, \mathfrak{N} \nmid 2 m$. Note that, in this case, since we assume that $\sqrt{\ell}, \sqrt{2 \ell}, \sqrt{3 \ell}$, $\sqrt{2 \ell \pm \sqrt{3} \ell} \notin F$, it follows that $a_{\ell}=0$ and $\tilde{F}=\mathbb{Q}(\sqrt{-\ell})$.

Remark 4.2. As we saw in Proposition 1.3, Theorem 1.4 and Corollary 3.8, we can often claim that $K=\mathbb{Q}(\sqrt{-N})$. When this is the case, last item in the statement of Theorem 4.1 can be removed and one can replace the sets $\mathcal{N}_{\ell}$ by the smaller sets $\mathcal{N}_{\ell}^{0}=\left\{\mathfrak{N}_{0} \mid \ell\right.$ or $\left.a^{2}-s \ell\right\}$, with $a$ and $s$ as in Definition 1.1. This is because in the above proof we have $\kappa(A, \mathfrak{N}) \mid 12$, thanks to Corollary 3.5.

Proof of Theorem 1.4. Since the hypothesis of Theorem $1.2(i i)$ are satisfied, we obtain that $N \equiv 3 \bmod 4$. 
Assume first that $M \equiv 3 \bmod 4$. Proposition 1.3 applies and asserts that $K \simeq$ $\mathbb{Q}(\sqrt{-d})$, where $d=M$ or $N$. Since $B \simeq\left(\frac{-d, M}{\mathbb{Q}}\right)$ we deduce that $d=N$ and $\left(\frac{-N}{M}\right)=-1$. Finally, for any odd prime $\ell$ such that $\left(\frac{\ell}{N}\right)=1$ and $N \notin \mathcal{N}_{\ell}$, Theorem 4.1 together with Gauss' reciprocity law asserts that $\left(\frac{-\ell}{M}\right)=-1$.

Assume now that $M \equiv 1 \bmod 4$. Proposition 1.3 now implies that $K \simeq \mathbb{Q}(\sqrt{-d})$ for $d=M N$ or $N$. In any case, it again follows as above that $\left(\frac{-N}{M}\right)=-1$.

If $d=N$, we similarly have $\left(\frac{-\ell}{M}\right)=-1$ for any prime $\ell$ such that $\left(\frac{\ell}{N}\right)=1$ and $N \notin \mathcal{N}_{\ell}$, even for $\ell=2$.

If $d=M N, N \equiv 3 \bmod 8$ by Lemma 3.4 and Corollary 3.8. Let $\ell \neq 2$ be a prime such that $N \notin N_{\ell}$ and $\left(\frac{-M N}{\ell}\right)=1$. Theorem 4.1 produces the congruences $\left(\frac{-\ell}{N}\right)=\left(\frac{-\ell}{M}\right)=-1$, which Gauss' reciprocity law shows to be incompatible with $\left(\frac{-M N}{\ell}\right)=1$.

\section{References}

[BFGR] N. Bruin, V. Flynn, J. Gonzàlez, and V. Rotger, On finiteness conjectures for endomorphism algebras of abelian surfaces, Math. Proc. Camb. Phil. Soc. 141:3 (2006), 383-408.

[Di] L. V. Dieulefait, Remarks on Serre's modularity conjecture, Preprint 2006.

[Ha] Y. Hasegawa, On some examples of modular QM-abelian surfaces, Proc. Japan Acad., Ser. A 72 (1996), 23-27.

[Jo] B.W. Jordan, Points on Shimura curves rational over number fields, J. Reine Angew. Math. 371 (1986), 92-114.

[KaWi] C. Khare and J.-P. Wintenberger, Serre's Modularity Conjecture (I), (II), Preprints 2006.

[Ki] M. Kisin, Modularity of 2-adic Barsotti-Tate representations, Preprint.

[Mi] J. S. Milne, Points on Shimura varieties mod p, Proc. Symp. Pure Math. 33 (1979), 165-184.

[PY] P. Parent and A. Yafaev, Proving the triviality of rational points on Atkin-Lehner quotients of Shimura curves, Math. Annalen 339:4 (2007), 915-935.

[Ri1] K. A. Ribet, Endomorphism algebras of abelian varieties attached to newforms of weight 2, Progress in Math. 12 (1981), 263-276.

[Ri2] - Abelian varieties over $\mathbb{Q}$ and modular forms, in Modular curves and abelian varieties, J. Cremona, J.-C. Lario, J. Quer, K. Ribet (eds.), Progress in Mathematics 224, Birkhäuser (2004), 241-261.

[Ro] V. Rotger, Rational points on Atkin-Lehner quotients of Shimura varieties, in preparation.

[RSY] V. Rotger, A. Skorobogatov, and A. Yafaev, Failure of the Hasse principle for Atkin-Lehner quotients of Shimura curves over $\mathbb{Q}$, Moscow Math. J. 5:2, (2005) 463-476.

[Se1] J.-P. Serre, Propriétés galoisiennes des points d'ordre finit des coubes elliptiques, Invent. Math. 15 (1972), 259-331.

[Se2] _ Quelques applications du théorème de densité de Chebotarev, Publ. I. H. É. S. 54 (1981), 123-201.

[SeTa] J.-P. Serre and J. Tate, Good reduction of abelian varieties, Ann. Math. 88 (1968), 492-517.

[Ta1] J. Tate, Endomorphisms of abelian varieties over finite fields, Invent. Math. 2 (1966), 134144.

[Ta2] Classes d'isogénie des variétés abéliennes sur un corps fini, Séminaire Bourbaki, 352 (1968-69), 95-110.

[Vi] M.F. Vignéras, Arithmétique des algèbres de quaternions, Lect. Notes Math. 800, 1980.

Universitat Politècnica de Catalunya, Departament de Matemàtica Aplicada IV (EPSeVG), Av. Victor Balaguer s/n, 08800 Vilanova i la Geltrú (Barcelona), Spain.

E-mail address: vrotger@ma4.upc.edu 